\title{
Intracranial temporal bone angiomatoid fibrous histiocytoma: illustrative case
}

\author{
Shivani Gillon, BS, BA, ${ }^{1}$ Jacqueline C. Junn, MD, ${ }^{6}$ Emily A. Sloan, MD, PhD, ${ }^{2}$ Nalin Gupta, MD, PhD, ${ }^{3}$ Alyssa Reddy, MD, ${ }^{4}$ and Yi Li, MD ${ }^{5}$
}

${ }^{1}$ School of Medicine, Departments of ${ }^{2}$ Pathology, ${ }^{3}$ Neurological Surgery, ${ }^{4}$ Neurology, and ${ }^{5}$ Radiology and Biomedical Imaging, University of California, San Francisco, California; and ${ }^{6}$ Department of Radiology, Mt. Sinai Medical Center, New York, New York

BACKGROUND Angiomatoid fibrous histiocytoma (AFH) is a rare, slowly progressive neoplasm that most commonly occurs in soft tissues. AFH rarely occurs in bone such as the calvaria. The authors present a case of AFH in the petrous temporal bone, which, to their knowledge, is the first case of AFH in this location.

OBSERVATIONS A 17-year-old girl presented with worsening positional headaches with associated tinnitus and hearing loss. Imaging demonstrated an extraaxial mass extending into the right cerebellopontine angle, with erosion of the petrous temporal bone, with features atypical for a benign process. The patient underwent retrosigmoid craniotomy for tumor resection. Pathology was consistent with a spindle cell tumor, and genetic testing further revealed an EWSR1 gene rearrangement, confirming the diagnosis of AFH. The patient was discharged with no complications. Her symptoms have resolved, and surveillance imaging has shown no evidence of recurrence.

LESSONS The authors report the first case of AFH in the petrous temporal bone and only the second known case in the calvaria. This case illustrates the importance of the resection of masses with clinical and imaging features atypical of more benign entities such as meningiomas. It is important to keep AFH in the differential diagnosis for atypical masses in the calvaria and skull base.

https://thejns.org/doi/abs/10.3171/CASE2026

KEYWORDS angiomatoid fibrous histiocytoma; temporal bone; pathology; radiology; EWSR1; meningioma

Angiomatoid fibrous histiocytoma (AFH) is a rare, slowly progressive neoplasm that occurs most frequently in children and young adults. ${ }^{1}$ The World Health Organization's classification of tumors of soft tissue and bone has characterized AFH as a slowgrowing and indolent tumor with a low risk of metastasis and recurrence. $^{2}$ The rate of metastasis is $1 \%-5 \%$ and warrants its classification as a low-grade malignant tumor. ${ }^{3}$ After initial treatment with complete, wide local excision, the recurrence is low. ${ }^{4}$ Patients with AFH may experience other systemic symptoms associated with tumor cytokine production, such as anemia, fever, and malaise. ${ }^{5}$

Thus far, the majority of reported cases of AFH have occurred in the superficial soft tissues, with relatively few reports in bone ${ }^{6}$ and even fewer with intracranial presentation. ${ }^{7-11}$ The rarity of AFH makes prospective diagnosis challenging, and the lesion has been mistaken for other pathologies, including angiosarcoma, eosinophilic granuloma, ${ }^{2,12}$ meningioma, ${ }^{10}$ aneurysmal bone cyst, ${ }^{13}$ or cavernous malformation. ${ }^{7}$ In this report, we present a rare case of primary AFH in the temporal bone, which, to our knowledge, is the first case of the histological diagnosis of AFH in this location.

\section{Illustrative Case \\ Clinical Presentation}

A 17-year-old girl with a past medical history of type 2 diabetes treated with metformin presented with new-onset daily positional headaches progressively worsening over a period of 6 months. The headaches were associated with high-pitched tinnitus, vertigo, vomiting, and blurry vision. Neurological examination was normal with the exception of a right esophoria corrected by cover testing and reduced deep tendon reflexes, likely related to poor glucose control. Audiogram demonstrated severe low-frequency loss in the right ear. Due to associated tinnitus and vertigo, magnetic resonance imaging (MRI) of the brain was performed to evaluate for a structural etiology for her symptoms.

ABBREVIATIONS AFH = angiomatoid fibrous histiocytoma; $\mathrm{CPA}=$ cerebellopontine angle; $\mathrm{CT}=$ computed tomography; $\mathrm{LCH}=\mathrm{Langerhans}$ cell histiocytosis; $\mathrm{MRI}=$ magnetic resonance imaging; NGS = next-generation sequencing.

INCLUDE WHEN CITING Published January 18, 2021; DOI: 10.3171/CASE2026.

SUBMITTED September 23, 2020. ACCEPTED September 29, 2020.

(C) 2021 The authors, CC BY-NC-ND 4.0 (http://creativecommons.org/licenses/by-nc-nd/4.0/), 

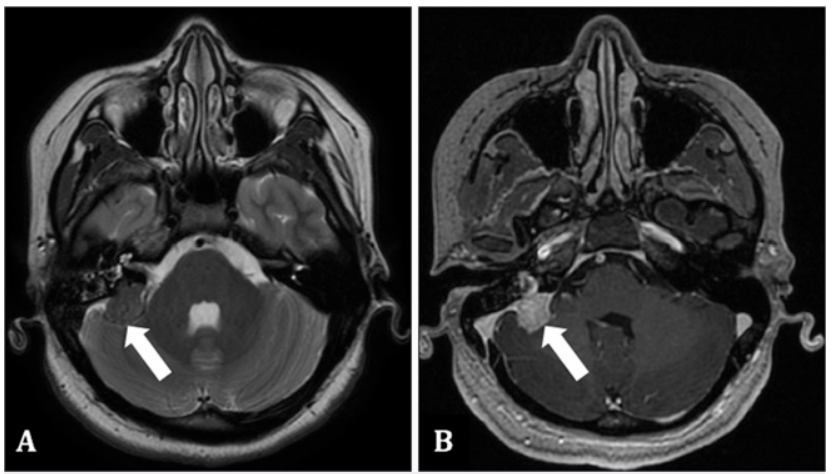

FIG. 1. A: Axial T2-weighted imaging demonstrates a heterogeneously T2 hypointense extraaxial mass in the right CPA, centered in the expected location of the endolymphatic sac, with extension through the mastoid temporal bone (arrow). There is mild mass effect on the adjacent right cerebellar hemisphere but no invasion. B: On axial T1 postcontrast imaging, the mass demonstrates avid postcontrast enhancement (arrow). No dural tail is visualized.

\section{Imaging}

MRI revealed a uniformly enhancing, extraaxial mass measuring up to $18 \mathrm{~mm}$ along the right petrous ridge, extending into the right cerebellopontine angle (CPA). The initial leading diagnostic consideration based on the MRI was a meningioma. This mass, however, demonstrated extension into the otic capsule (Fig. 1) and the lack of a dural tail, which are atypical features for a meningioma. Because of these unusual imaging findings, a computed tomography (CT) scan was obtained to evaluate the temporal bone. CT of the temporal bone showed that the mass was eroding into the adjacent petrous temporal bone, the lateral semicircular canal, and protruded into the middle ear cavity (Fig. 2). The involvement of the inner ear likely contributed to the patient's symptoms of vertigo and tinnitus. Based on this CT appearance, the potential diagnoses were expanded to include an endolymphatic sac tumor or Langerhans cell histiocytosis (LCH).

\section{Operation}

Based on the presence of symptoms and imaging features suggestive of a possible neoplasm, a resection of the mass was recommended.

The patient underwent a right retrosigmoid craniotomy for microsurgical resection of the tumor, followed by a titanium mesh repair of the calvarial defect. The vestibular aqueduct was noted to be widened by the tumor, and the tumor was successfully removed from the endolymphatic duct. When dissected, the tumor was fibrous and calcific, and the frozen section was consistent with a spindle cell tumor.

\section{Pathology}

Pathology demonstrated a nodular proliferation of epithelioid tumor cells with irregular nuclear contours, vesicular chromatin, and indistinct nucleoli (Fig. 3) with occasional whorl-like growth patterns. Tumor nodules were separated by dense fibrous bands containing robust lymphoplasmacytic inflammatory infiltrates with germinal center formation. Mitoses were difficult to find, and there was no necrosis. Additionally, tumor cells showed patchy positivity for desmin, CD99, epithelial membrane antigen, and CD68, with focal S100. Furthermore, tumor cells were negative for SMA, CD1a, SSTR2a, and ALK.

The specimen was further subjected to genetic testing. EWSR1 breakapart testing by fluorescence in situ hybridization revealed an EWSR1 gene rearrangement. Next-generation sequencing (NGS) using the

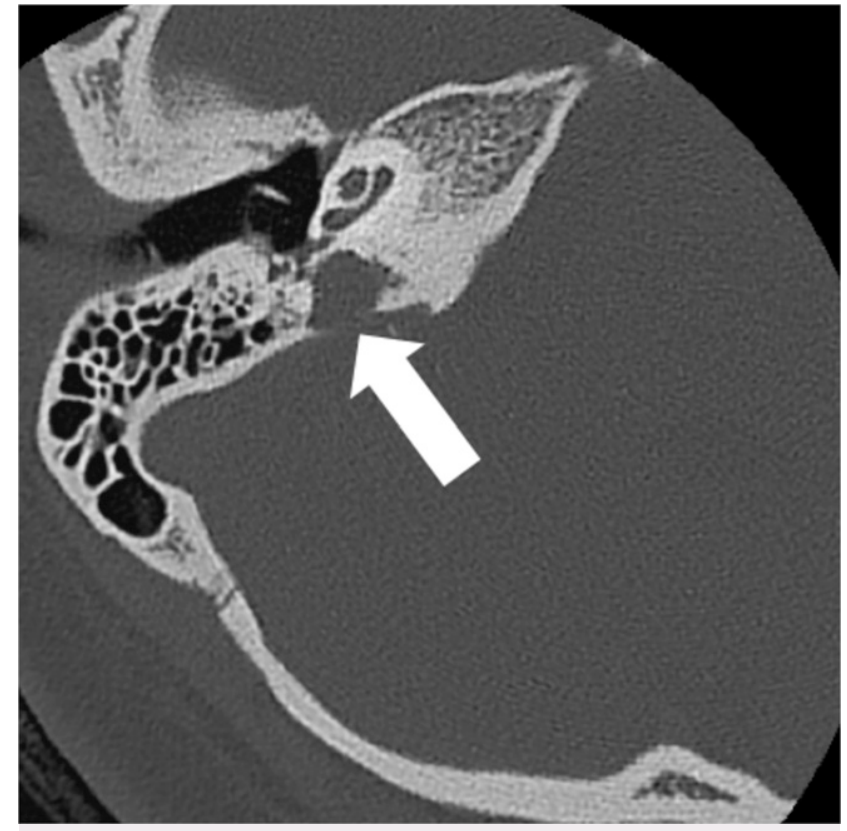

FIG. 2. Axial high-resolution CT of the right temporal bone demonstrates osseous erosion of the underlying temporal bone (arrow). The erosion extends through the posterior semicircular canal to the middle ear.

UCSF500 Cancer Gene panel identified an EWSR1 gene rearrangement on chromosome 22q12. Sequencing reads were obtained over the reciprocal fusion between EWSR1 and intron 1-2 of the LIMA1 gene, which is located immediately adjacent to the ATF1 gene on chromosome 12q13, raising the likely possibility of an in-frame EWSR1-ATF1 fusion. Finally, the presence of EWSR-ATF1 fusion together with morphological, histological, and immunological findings supported the diagnosis of $\mathrm{AFH}$.

\section{Clinical Follow-Up}

The patient was discharged a week after the resection without complications. Since discharge, the patient's headaches have subsided and hearing loss in the right ear has resolved. Follow-up MRI scans obtained 3 months and 6 months after surgery demonstrated no evidence of recurrence.

\section{Discussion}

\section{Observations}

AFH is a rare, indolent neoplasm that is often confused with other entities. Previous instances of AFH in bone have been described in both the radiology and pathology literature. ${ }^{13} \mathrm{AFH}$ in the skull is quite rare, with only one other reported case of AFH in the parietal bone. ${ }^{12}$ In this case, AFH was preoperatively presumed to represent an eosinophilic granuloma, and pathological and molecular analysis of the resected tumor tissue was required to ultimately confirm the diagnosis of $\mathrm{AFH} .{ }^{12}$

In our case, the imaging characteristics of the tumor initially pointed toward the diagnoses of meningioma, endolymphatic sac tumor, or $\mathrm{LCH}$. On postcontrast T1-weighted MRI, meningiomas demonstrate avid enhancement, as in our case. However, meningiomas often demonstrate a dural tail, which was absent in our case, and meningiomas are more frequently associated with hyperostosis of the underlying bone, not erosion. Demographically, meningiomas most commonly occur during the 3rd to 6th decades of life and are relatively rare in children and adolescents. ${ }^{14,15}$ 


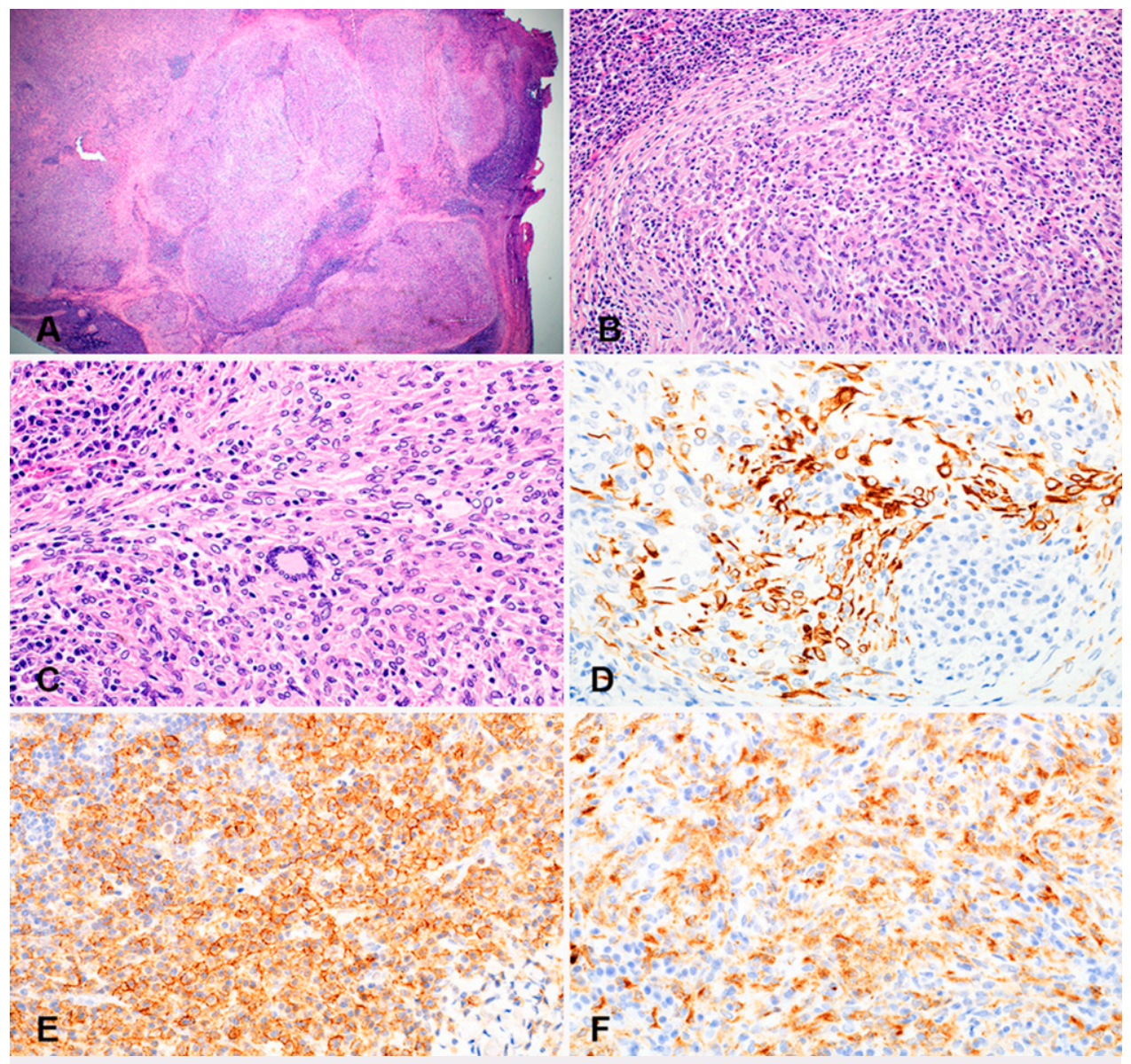

FIG. 3. Histological and immunohistochemical features. A: Hematoxylin-eosin stain demonstrates a solidappearing neoplasm composed of nodules of tumor cells separated by fibrous bands and surrounded by a fibrous pseudocapsule. There is a robust lymphoplasmacytic inflammatory infiltrate (original magnification $\times 20$ ). Higher power views ( $B$, original magnification $\times 200$; and $C$, original magnification $\times 400$ ) show nodules of epithelioid tumor cells with ovoid to vesicular nuclei, coarse chromatin, and dense eosinophilic cytoplasm, forming a vaguely whorled architectural pattern. There are scattered lymphocytes and plasma cells within the tumor nodules, which are also surrounded by dense lymphoplasmacytic inflammation. Rare Touton-like giant cells are present. Tumor cells show strong, patchy positivity for desmin (D), epithelial membrane antigen $(E)$, and CD68 (F) (original magnifications $\times 400$ ).

Moreover, although the mass was located in the region of the endolymphatic sac, our case showed darker T2 signal characteristics than expected for endolymphatic sac tumors. Furthermore, endolymphatic sac tumors also characteristically cause permeative bone destruction instead of frank erosion. ${ }^{16} \mathrm{LCH}$ tends to occur in the pediatric population and is commonly found in flat bones, particularly the calvaria and temporal bones, which would be consistent with this case. ${ }^{17}$ On CT, however, LCH lesions commonly demonstrate a punched-out, beveled appearance as a result of the asymmetrical destruction of the inner and outer plates of the calvaria. ${ }^{18}$ The erosive changes of the temporal bone in this case are unusual for $\mathrm{LCH}$. The diagnosis of AFH was not prospectively considered in this case because of its relative rarity and lack of previous description in this location.

The common histological features in AFH include sheets of spindle, ovoid, or round cells with pale, vesicular nuclei. ${ }^{19}$ These sheets often become hemorrhagic and can lead to the creation of fluid or blood-filled pseudoangiomatous spaces, which can compose a major part of the tumor. Four histological features of AFH have been described by Ochalski et al., which include 1) fibrous pseudocapsule; 2) cellular foci of pleomorphic, hyperchromatic, histiocytoid cells; 3) focal areas of hemorrhage and blood-filled spaces lacking endothelial lining; and 4) lymphohistiocytic inflammatory cuff surrounding the lesion. ${ }^{7}$

Molecular hallmarks of AFH are still under investigation and not yet exhaustively available due to the paucity of cases and reported specific gene fusions. Thus far, AFH has been linked to three cytogenetic translocations- $\mathrm{t}(2 ; 22)(\mathrm{q} 33 ; \mathrm{q} 12), \mathrm{t}(12: 22)(\mathrm{q} 13: q 12)$, and $\mathrm{t}(12: 16)(\mathrm{q} 13$ : p11) -which correspond to EWSR1/CREB1, EWSR1/ATF1, and FUS/ATF1 gene fusions, respectively. ${ }^{13}$ Of these gene fusions, EWSR1/CREB1 is the most predominant and can be used to confirm the diagnosis of $\mathrm{AFH}{ }^{20}$

However, not all gene fusions are limited to one pathology and may overlap with other diagnoses. Therefore, these fusions alone may not be sufficient for an accurate diagnosis. For example, one notable overlap involves intracranial AFH and more recently described intracranial myxoid mesenchymal tumors. Like AFH, myxoid mesenchymal tumors are more commonly found in children and young adults and demonstrate similar EWSR1-CREB family fusions. ${ }^{19}$ However, intracranial myxoid mesenchymal tumors were suggested to represent a distinct tumor 
type because of their intracranial location and histological appearance, combined with the lack of several morphological features typically associated with AFH such as the fibrous pseudocapsule, hemorrhagic spaces, and surrounding lymphoplasmacytic cuffs. Currently, controversy remains regarding whether intracranial myxoid mesenchymal tumors are unique entities or whether they represent intracranial variants of AFH. ${ }^{21}$ Nevertheless, a multifaceted approach using imaging, molecular studies, and pathological findings will facilitate further understanding of these intracranial mesenchymal tumors with EWSR-CREB fusions and promote appropriate therapy. These examples highlight the importance of utilizing genetic information in concert with other morphological and histological findings to determine the correct diagnosis. $^{22}$

In this patient, given the nonspecific imaging findings, the NGS cancer panel greatly assisted with arriving at a diagnosis by identifying a likely EWSR-ATF1 gene fusion, a molecular feature of AFH. ${ }^{13}$ Furthermore, one notable feature of this case was the focal $\mathrm{S} 100$ positivity, which is typically seen in LCH and Rosai-Dorfman disease. Therefore, these findings illustrate that, for soft tissue tumors like AFH, molecular and immunohistochemical markers may offer the most information in assisting appropriate differential pathologies and, ultimately, the greatest accuracy for diagnosis.

\section{Lessons}

We report the first case of intracranial AFH associated with the petrous temporal bone and only the second known case involving the calvaria. The radiological appearance of AFH is not distinct and carries a differential diagnosis, but this disease entity should be considered for intracranial soft tissue tumors that appear to arise from dura and involve bone. In a patient with concerning symptoms and imaging features atypical for more benign and common processes such as meningiomas, resection and pathology are indicated for more definitive diagnosis. Additionally, aggregating data from multiple diagnostic modalities, including imaging, immunohistochemical staining, and molecular genetics, can assist in an accurate final diagnosis.

\section{References}

1. Fan $Q$, Allen $P W$. Angiomatoid malignant fibrous histiocytoma. Zhonghua Bing Li Xue Za Zhi. 1996;25(1):30-32.

2. Fletcher CDM. The evolving classification of soft tissue tumours: an update based on the new WHO classification. Histopathology. 2006;48(1):3-12.

3. Fanburg-Smith JC, Miettinen M. Angiomatoid "malignant" fibrous histiocytoma: a clinicopathologic study of 158 cases and further exploration of the myoid phenotype. Hum Pathol. 1999;30(11): 1336-1343.

4. Costa MJMD, Weiss SW. Angiomatoid malignant fibrous histiocytoma. A follow-up study of 108 cases with evaluation of possible histologic predictors of outcome. Am J Surg Pathol. 1990;14(12):1126-1132.

5. Thway K, Fisher C. Angiomatoid fibrous histiocytoma: the current status of pathology and genetics. Arch Pathol Lab Med. 2015; 139(5):674-682.

6. Chen G, Folpe AL, Colby TV, et al. Angiomatoid fibrous histiocytoma: unusual sites and unusual morphology. Mod Pathol. 2011; 24(12):1560-1570.

7. Ochalski PG, Edinger JT, Horowitz MB, et al. Intracranial angiomatoid fibrous histiocytoma presenting as recurrent multifocal intraparenchymal hemorrhage. J Neurosurg. 2010;112(5):978-982.

8. Alshareef HMA, Alhasan AHA, Felemban MG, et al. Angiomatoid fibrous histiocytoma in the forearm: a case report. Cancer Clin Oncol. 2016;6(1):36.

9. Dunham C, Hussong J, Seiff M, et al. Primary intracerebral angiomatoid fibrous histiocytoma: report of a case with a $\mathrm{t}(12$; 22)(q13;q12) causing type 1 fusion of the EWS and ATF-1 genes. Am J Surg Pathol. 2008;32(3):478-484.

10. Hansen JM, Larsen VA, Scheie $D$, et al. Primary intracranial angiomatoid fibrous histiocytoma presenting with anaemia and migraine-like headaches and aura as early clinical features. Cephalalgia. 2015;35(14):1334-1336.

11. Spatz M, Nussbaum ES, Lyons L, et al. Primary intracranial angiomatoid fibrous histiocytoma: a case report and literature review. Br J Neurosurg. 2018;0(0):1-3.

12. Zheng $X$, Han FG, Luo L, et al. Angiomatoid fibrous histiocytoma mimicking eosinophilic granuloma in a pediatric patient. World Neurosurg. 2019;129:345-348.

13. Petrey WB, LeGallo RD, Fox MG, et al. Imaging characteristics of angiomatoid fibrous histiocytoma of bone. Skeletal Radiol. 2011; 40(2):233-237.

14. Wiemels J, Wrensch M, Claus EB. Epidemiology and etiology of meningioma. J Neurooncol. 2010;99(3):307-314.

15. Kotecha RS, Pascoe EM, Rushing EJ, et al. Meningiomas in children and adolescents: a meta-analysis of individual patient data. Lancet Oncol. 2011;12(13):1229-1239.

16. Bonneville F, Sarrazin JL, Marsot-Dupuch K, et al. Unusual lesions of the cerebellopontine angle: a segmental approach. Radiographics. 2001;21(2):419-438.

17. Azouz EM, Saigal G, Rodriguez MM, et al. Langerhans' cell histiocytosis: pathology, imaging and treatment of skeletal involvement. Pediatr Radiol. 2005;35(2):103-115.

18. Zheng H, Xia Z, Cao W, et al. Pediatric Langerhans cell histiocytosis of the temporal bone: clinical and imaging studies of 27 cases. World J Surg Oncol. 2018;16(1):72.

19. Bale TA, Oviedo A, Kozakewich $H$, et al. Intracranial myxoid mesenchymal tumors with EWSR1-CREB family gene fusions: myxoid variant of angiomatoid fibrous histiocytoma or novel entity? Brain Pathol. 2018;28(2):183-191.

20. Antonescu CR, Cin PD, Nafa K, et al. EWSR1-CREB1 is the predominant gene fusion in angiomatoid fibrous histiocytoma cristina. Genes, Chromosom Cancer. 2007;46(12): 1051-1060.

21. Gareton A, Pierron G, Mokhtari K, et al. ESWR1-CREM fusion in an intracranial myxoid angiomatoid fibrous histiocytoma-like tumor: A case report and literature review. J Neuropathol Exp Neurol. 2018;77(7):537-541.

22. Kao YC, Sung YS, Zhang L, et al. EWSR1 fusions with CREB family transcription factors define a novel myxoid mesenchymal tumor with predilection for intracranial location. Am J Surg Pathol. 2017;41(4):482-490.

\section{Disclosures}

Dr. Gupta receives financial support from Y-mAbs Therapeutics and Encoded Therapeutics for work outside of this study.

\section{Author Contributions}

Conception and design: Li. Acquisition of data: Li, Junn, Sloan, Reddy. Analysis and interpretation of data: Li, Junn, Sloan, Gupta, Reddy. Drafting the article: Li, Gillon, Junn. Critically revising the article: all authors. Reviewed submitted version of manuscript: all authors. Approved the final version of the manuscript on behalf of all authors: Li. Study supervision: Li.

\section{Supplemental Information}

Previous Presentations

Material related to this work was presented at the 2020 American Society of Head and Neck Radiology virtual meeting held September 9-13, 2020.

\section{Correspondence}

Yi Li: University of California, San Francisco, CA. yi.li@ucsf.edu. 